\title{
INVERTIR EN BOLSA: EXPECTATIVAS, VOLATILIDAD Y GANANCIAS
}

Pablo Mauricio Pachas"

E-mail: pwmauricio@yahoo.es

\begin{abstract}
RESUMEN
Se presenta a la Bolsa de Valores de Lima como una importante y lucrativa alternativa para los inversionistas, asimismo se destaca que en los últimos cinco años la rentabilidad promedio anual ha superado el 80\%, convirtiéndose en la Bolsa más rentable del mundo en el 2006.

En ese sentido la marcada volatilidad por la influencia del mercado externo produce cambios marcados en las cotizaciones de las principales acciones que cotizan en el mercado internacional; en ese contexto se debe entender a este mercado como inversiones de largo plazo, al que se deben destinar recursos no prioritarios para el inversionista.
\end{abstract}

Palabras clave: Volatilidad en bolsa de valores.

\begin{abstract}
This article expresses the importance and lucrative alternative for the investors in the Stock Exchange of Lima. In the last five years, the average of profitability per year has overcome the $80 \%$ until being ended up transforming into the 2006 in the most profitable Stock Exchange in the world.

In that sense the marked volatility for the influence of the external market that produces changes marked in the rates of the main actions that they quote in the international market, in that context he/she should understand each other to this market as investments of long term and that non highpriority resources should be dedicated for the investor.
\end{abstract}

Key words: Volatility in Stock Exchange.

\footnotetext{
* Magíster en Economía y con Estudios de Doctorado en Ciencias Empresariales. Postgrado en Finanzas-ESAN. Economista. Asesor Financiero y Consultor del Programa de Naciones Unidas para el Desarrollo (PNUD). Consultor de Negocios Bursátiles y Director de la Escuela de Administración de Negocios Internacionales de la Facultad de Ciencias Administrativas de la Universidad Nacional Mayor de San Marcos.
} 


\section{INTRODUCCION}

La Bolsa de Valores de Lima ha evidenciado ser una importante y lucrativa alternativa para los inversionistas que apostaron por ella, al alcanzar en los últimos cinco años la elevada rentabilidad del $83 \%$ en promedio anual (variación del ISBVL), llegando a convertirse en el 2006 en la Bolsa más rentable del mundo.

No obstante la tendencia alcista que mantuvo hasta el tercer trimestre de este año 2007, se ha visto orientada drásticamente hacia la baja por la marcada volatilidad del mercado externo, produciendo pánico y en consecuencia la salida significativa de los pequeños inversionistas. Este comportamiento nos lleva a reflexionar sobre la importancia de informarse de lo que significa invertir en bolsa, por lo que considero importante tratar de explicar el qué, cómo y el por qué del funcionamiento de este mercado, conceptos que desarrollaré a continuación y que espero aclaren dudas y ayuden a los futuros inversionistas a tomar mejores decisiones.

En primer término es necesario saber qué es el Mercado de Valores. Es aquel donde se negocian valores mobiliarios, entendiéndose como tales a aquellos valores emitidos por empresas públicas o privadas, gobiernos e instituciones en forma masiva y libremente negociable, que confieren a sus titulares derechos crediticios (bonos) o de participación en el patrimonio o utilidades del emisor (acciones), pudiendo ser estos bonos, acciones, ADR's, certificados de participación, certificados de suscripción preferente, instrumentos de corto plazo, etc. En primera instancia la negociación de las emisiones se realiza en el mercado primario; luego las que son autorizadas por la Comisión Nacional Supervisora de Empresas y Valores (CONASEV) pasan a ser negociadas al mercado secundario o Bolsa de Valores.

El mercado primario es donde los emisores acuden para financiarse a través de la colocación de valores de primera emisión, ya sea mediante bonos (renta fija) o acciones (renta variable), los que deben cumplir con siguientes requisitos:

- Tener el perfil adecuado como para convencer a los inversionistas que le entreguen sus recursos. Para ello el emisor debe brindar la información que el inversionista necesita para tomar decisiones, lo que incluye la evolución de los resultados de la empresa que demuestren que tiene perspectivas de crecimiento futuro, y que por tanto será conveniente invertir en esa empresa.

- Estar dispuesto a difundir información al mercado. Toda empresa debe entregar sus estados financieros trimestrales no auditados y anuales auditados, memoria y hechos de importancia para que se conviertan en información pública, es decir información disponible para todos los inversionistas.

- Sus necesidades de financiamiento deben ser de la magnitud necesaria que permita diluir los costos de transacción del mercado de valores. En el caso de los instrumentos de deuda, además de la tasa de rendimiento ofrecida a los inversionistas, las empresas deben asumir una serie de costos fijos como los costos de estructuración, colocación, representante de obligacionistas y clasificación de riesgo. Si el monto de colocación es muy pequeño, no se justificarían los costos. En la actualidad los costos de transacción en el mercado de valores peruano sólo justifican emisiones mayores a US\$ 10 millones (para empresas que ofrecen un riesgo conservador); ésta es la principal razón por la cual las pequeñas empresas no pueden acceder al mercado de valores.

La importancia de este mercado en nuestro país se ve claramente reflejada en el Cuadro $\mathrm{N}^{\circ} 1 \mathrm{y}$ Gráfico $\mathrm{N}^{\circ} 1$, donde podemos apreciar que el mayor dinamismo se produjo a partir del 2003 con el nivel de valores autorizados-inscritos de US\$1,759 millones, elevándose a US\$ 3,084 millones en el 2006. Sin embargo hay que advertir la notoria sobreoferta que ocasionó la disminución en las colocaciones (baja demanda de valores inscritos) como la de menos $39 \%$ en el 2004, descendiendo aún mas en el 2006 en menos 49\%, clara evidencia de la desconfianza por el riesgo percibido por parte de los inversionistas y, en consecuencia, su escasa contribución al mercado de valores.

La Bolsa de Valores es el mercado donde se centraliza la negociación de valores previamente emitidos y colocados, permitiendo a los tenedores de valores venderlos y obtener liquidez como también facilitar el ingreso de compradores o inversionistas a adquirir valores a precios o cotización determinados por el libre juego de la oferta y la demanda; de allí que se constituye en alternativa de inversión expuesta a los riesgos y expectativas que experimenta este mercado por factores externos e internos, comportamiento que conlleva a que se le denomine termómetro de la economía. 
Cuadro N. ${ }^{\circ}$. Valores inscritos vs. colocados por oferta pública primaria.

\begin{tabular}{|c|c|c|c|}
\hline US\$MM & Inscrito & Colocado & $\begin{array}{c}\text { Colocado/ } \\
\text { Inscrito }\end{array}$ \\
\hline 1992 & 10 & 10 & 1 \\
1993 & 20 & 20 & 1 \\
1994 & 95 & 92 & 0,97 \\
1995 & 340 & 315 & 0,93 \\
1996 & 849 & 770 & 0,91 \\
1997 & 1664 & 1376 & 0,84 \\
1998 & 1092 & 590 & 0,54 \\
1999 & 817 & 569 & 0,7 \\
2000 & 1539 & 1014 & 0,66 \\
2001 & 1867 & 1096 & 0,59 \\
2002 & 1218 & 828 & 0,759 \\
2003 & 1759 & 1451 & 0,82 \\
2004 & 2060 & 1248 & 0,61 \\
2005 & 2086 & 1474 & 0,71 \\
2006 & 3084 & 1565 & 0,51 \\
\hline
\end{tabular}

Gráfico $\mathrm{N}^{\circ}$ 1. Valores inscritos vs. colocados por oferta pública primaria.

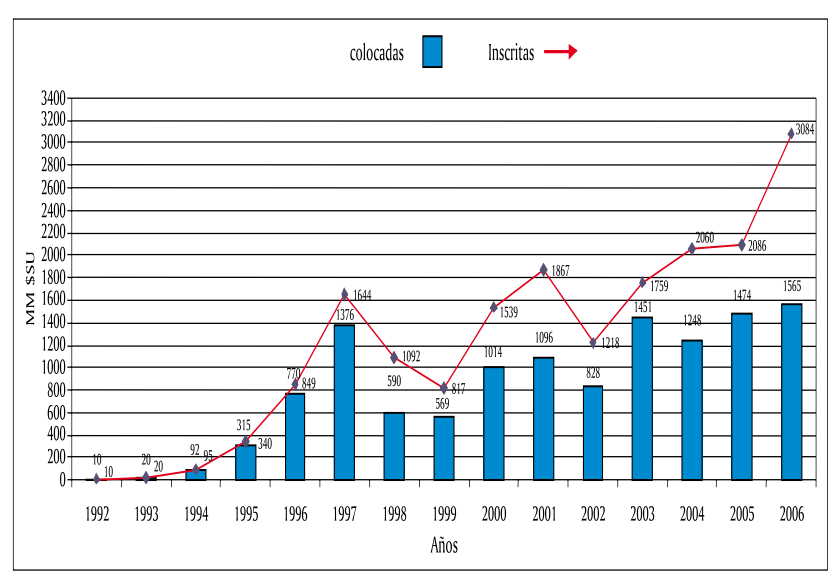

Este mercado desarrolla sus operaciones a través del mercado bursátil y extrabursátil. El mercado extrabursátil es donde se negocian valores fuera de la Bolsa de Valores, como Warrants, aceptaciones bancarias, certificados $\mathrm{BCR}$, etc.; también es llamado mercado sobre el mostrador.

El mercado bursátil es administrado por la Bolsa de Valores de Lima, donde se negocian valores a través de agentes especializados llamados Sociedades Agentes de Bolsa (SAB), quienes representan a los inversionistas y ejecutan las órdenes de compra y venta. Existen dos mecanismos centralizados de negociación:
- Mecanismo de Instrumentos de Emisión No Masiva (MIENM).

- Rueda de Bolsa: Donde se realizan negociaciones de valores mediante operaciones al contado, operaciones de reporte y day trade (en el día).

Entre los objetivos de la Bolsa se destaca el facilitar el intercambio entre las entidades que requieren financiación y los inversores que proporcionan liquidez. También el de proporcionar liquidez a los titulares o tenedores de valores en Bolsa y con esto fijar los precios de los títulosvalores a través de la ley de la oferta y la demanda, y tener información sobre las empresas que cotizan en Bolsa, con la intención de publicar los precios y cantidades negociables para informar a los inversores y entidades interesados.

\section{Beneficios y riesgos}

Como en toda decisión de inversión, lo importante es poder medir los beneficios y riesgos que esta conlleva. Para los inversionistas, los beneficios en la Bolsa de Valores son el obtener rendimientos mayores que en el sistema bancario; sin embargo la exposición al riesgo es mayor, siendo necesario para ello sustentar las decisiones de inversión en la diversificación del riesgo. Los inversionistas institucionales pueden acceder a montos importantes de financiamiento. Los riesgos para los inversionistas son que no existe un rendimiento asegurado por sus inversiones en valores como en el caso de los depósitos en banco, ya que debido al riesgo del mercado podría abrir o bajar los precios de los valores; en gran o poca medida el riesgo crediticio del emisor puede disminuir y afectar su capacidad de pago.

Para los emisores, los beneficios son obtener un menor costo de financiación en comparación con los bancos y esto estará relacionado con la cantidad de acciones que emitan. Según las necesidades de financiación de deuda también tienen la opción de elegir si desean trabajar a corto o mediano plazo utilizando los bonos y otros instrumentos de corto plazo, o emitir acciones con derecho o sin derecho a voto, en soles o dólares.

Existe una demanda potencial de inversionistas institucionales como los fondos privados de pensiones, fondos mutuos, compañías de seguros, bancos y la Oficina de Normalización Provisional (ONP), que acumulan inversiones por más de US\$ 30,000 millones. Los riesgos para los emisores radican en 
el peligro de pérdida de control ya que si se emiten demasiadas acciones con derecho a voto los accionistas fundadores podrían perder el control de la empresa. Este peligro se puede minimizar emitiendo acciones Tipo B (sin derecho a voto).

En cuanto al desempeño de la Bolsa de Valores, éste se puede apreciar a través de las variaciones de las cotizaciones de las acciones, expresadas en la representación de los índices bursátiles. Los cuatro índices que miden el pulso de la Bolsa de Valores de Lima son:

- Índice General de la Bolsa de Valores de Lima (IGBVL). Refleja la tendencia promedio de las cotizaciones de las 33 acciones más negociadas en la Bolsa de Valores. Su cálculo considera las variaciones de las acciones, cuya fecha base es el 30 de diciembre de $1991=100$.

- Índice Selectivo de la Bolsa de Valores de Lima (ISBVL). Mide las variaciones de las cotizaciones de las quince acciones más representativas de la Bolsa de Valores de Lima. Al igual que el IGBVL, la base es la fecha del 30 de diciembre de $1991=100$.

- Índice Selectivo Perú-15. Mide el comportamiento de las quince acciones más negociadas de empresas locales, es decir aquellas que registren la mayor parte de sus actividades en el Perú. Por lo tanto, este indicador tiene una mayor correlación con el desarrollo de la economía peruana al no considerar valores extranjeros (que no registran sus principales actividades en el país) en su cartera. Dado que el actual ISBVL está conformado por acciones locales, el ISP-15 tiene la misma cartera pero esto no es una situación permanente, sólo se ha dado el caso de que las quince acciones más representativas sean aquellas que tengan la mayor parte de sus actividades en el Perú.

- Índice Nacional de Capitalización (INCA). Es un índice que mide el comportamiento de las veinte acciones peruanas más líquidas negociadas en la BVL pero a diferencia de los tres índices anteriores, el índice INCA no se calcula en base a la liquidez de las acciones sino en base al tamaño del patrimonio de la empresa representado en la Bolsa (free float); esto significa que a mayor nivel de capitalización bursátil recibe un mayor peso, por lo que no utiliza pesos fijos sino pesos que varían automáticamente. En consecuencia, permite la comparación más eficaz con los principales índices bursátiles del mundo que cuentan con una metodología muy similar.

\section{Rentabilidad de los últimos seis años}

La rentabilidad es descrita por las variaciones en los precios de los acciones que se negocian en estos mercados de la bolsa, apreciándose en los índices anteriormente comentados. Sin embargo, los que explican el comportamiento general se expresan a través de las variaciones del IGBVL, que comprende a la cartera de las 33 principales acciones y la variación del ISGBVL, que comprende a las quince de mayor importancia, denominadas también las "blue chip".

En el Gráfico $N^{\circ}$ 2: Rentabilidad Bursátil, se describe la variación del IGBVL e ISBVL, y se aprecia que la rentabilidad se inicia en 2002 en $18 \%$ (IGBVL) y $15 \%$ (ISBVL), para luego pasar a la impresionante rentabilidad alcanzada en el 2006 , que alcanza el $168 \%$ (IGBVL) y $188 \%$ (ISBVL).

Gráfico $N^{\circ}$ 2. BVL: Rentabilidad Bursátil (Var. \% de los índices de la BVL).

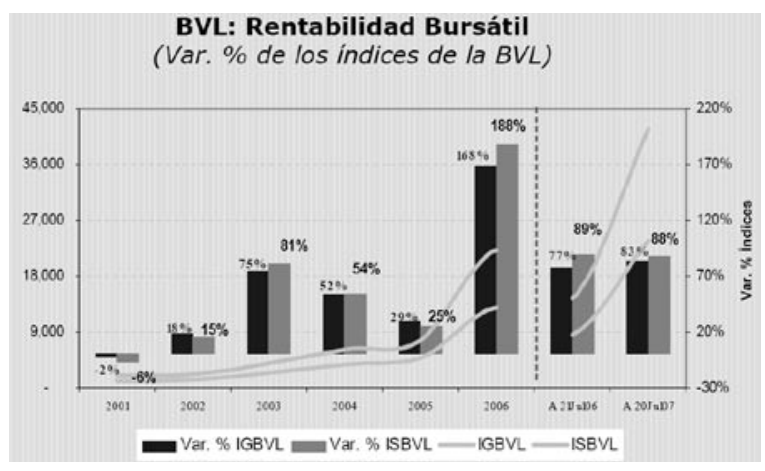

La rentabilidad de la Bolsa iniciada en el 2002 con $18.32 \%$ (IGBVL) como se puede observar en el Cuadro $\mathrm{N}^{\circ} 2$ : Índice de Cotizaciones, está relacionada con los beneficios del mercado internacional, particularmente por el alza en los minerales que elevó impresionantemente los ingresos de las empresas mineras dedicadas a la exportación, y conllevó a incrementar los saldos favorables en la Balanza Comercial, situación favorable del comercio exterior que se puede apreciar en el superávit en la Balanza Comercial, iniciado también en el 2002, lo que vemos detallado en el Gráfico $\mathrm{N}^{\circ} 3$ : Evolución de la Balanza Comercial y proyecciones. 
Cuadro $\mathrm{N}^{\circ}$ 2. Índice de cotizaciones.

\begin{tabular}{lcccccc}
\hline \multicolumn{1}{c}{ Base 30/12/1991=100 } & $\mathbf{2 0 0 1}$ & $\mathbf{2 0 0 2}$ & $\mathbf{2 0 0 3}$ & $\mathbf{2 0 0 4}$ & $\mathbf{2 0 0 5}$ & $\mathbf{2 0 0 6}$ \\
\hline & & & & & & \\
Índice general (IGBVL) & $1,176.45$ & $1,391.97$ & $2,435.04$ & $3,710.39$ & $4,802.25$ & $12,884.20$ \\
Variación nominal \% & $-2.64 \%$ & $18.32 \%$ & $74.93 \%$ & $52.37 \%$ & $29.43 \%$ & $168.30 \%$ \\
Variación real \% & $-2.52 \%$ & $16.80 \%$ & $70.70 \%$ & $47.25 \%$ & $27.53 \%$ & $165.28 \%$ \\
Variación en US\$ \% & $0.24 \%$ & $16.28 \%$ & $76.34 \%$ & $60.51 \%$ & $24.56 \%$ & $186.92 \%$ \\
Máxima & $1,418.44$ & $1,406.98$ & $2,452.08$ & $3,710.39$ & $5,235.65$ & $13,143.57$ \\
Fecha & 5 -jun. & 12 -dic. & 29 -dic. & 30 -dic. & 9 -dic. & 18 -dic. \\
Mínima & $1,131.21$ & $1,109.89$ & $1,405.21$ & $2,493.81$ & $3,703.17$ & $4,602.95$ \\
Fecha & 6 -nov. & 3 -jul. & 2 -ene. & 2 -ene. & 13 -may. & 13 -ene. \\
& & & & & & \\
Índice selectivo (ISBVL) & $1,917.16$ & $2,202.32$ & $3,993.86$ & $6,159.63$ & $7,681.11$ & $22,159.28$ \\
Variación nominal \% & $-6.32 \%$ & $14.87 \%$ & $81.35 \%$ & $54.23 \%$ & $24.70 \%$ & $188.49 \%$ \\
Variación real \% & $-6.20 \%$ & $13.35 \%$ & $76.96 \%$ & $49.04 \%$ & $22.87 \%$ & $185.24 \%$ \\
Variación en US\$ \% & $-3.55 \%$ & $12.89 \%$ & $82.81 \%$ & $62.46 \%$ & $20.01 \%$ & $208.52 \%$ \\
Máxima & $2,402.08$ & $2,224.28$ & $4,035.68$ & $6,159.63$ & $8,422.51$ & $23,099.57$ \\
Fecha & 5 -jun. & 13 -dic. & 29 -dic. & 30 -dic. & 9 -dic. & 18 -dic. \\
Mínima & $1,824.04$ & $1,742.68$ & $2,227.26$ & $4,107.85$ & $6,081.55$ & $7,433.65$ \\
Fecha & 10 -oct. & 3 -jul. & 2 -ene. & 2 -ene. & 13 -may. & 13 -ene. \\
& & & & & & \\
\hline
\end{tabular}

Cuadro $\mathrm{N}^{\circ} 3$. Rendimientos Principales Índices Bursátiles 2004-2007 (variaciones porcentuales de cada período).

\begin{tabular}{lcccc} 
& $\mathbf{2 0 0 4}$ & $\mathbf{2 0 0 5}$ & $\mathbf{2 0 0 6}$ & $\mathbf{2 0 0 7}$ \\
\cline { 2 - 5 } & $70 \%$ & $18 \%$ & $\mathbf{2 1 6 \%}$ & $\mathbf{5 2 \%}$ \\
Perú selectivo 15 & $61 \%$ & $22 \%$ & $\mathbf{1 8 7 \%}$ & $\mathbf{4 8 \%}$ \\
Perú IGVBL & $63 \%$ & $17 \%$ & $\mathbf{2 0 9 \%}$ & $\mathbf{4 5 \%}$ \\
Perú ISBVL & $-15 \%$ & $-6 \%$ & $\mathbf{1 3 8 \%}$ & $30 \%$ \\
China Shangai & $29 \%$ & $20 \%$ & $34 \%$ & $13 \%$ \\
Chile IPSA & $28 \%$ & $\mathbf{4 9 \%}$ & $45 \%$ & $12 \%$ \\
Brasil Bovespa & $16 \%$ & $11 \%$ & $35 \%$ & $11 \%$ \\
Alemania DAX & $48 \%$ & $44 \%$ & $45 \%$ & $10 \%$ \\
México Mexbol & $16 \%$ & $8 \%$ & $30 \%$ & $6 \%$ \\
Francia CAC & $16 \%$ & $6 \%$ & $26 \%$ & $4 \%$ \\
UK FTSE & $9 \%$ & $2 \%$ & $10 \%$ & $3 \%$ \\
USA Nasdaq & $13 \%$ & $22 \%$ & $6 \%$ & $3 \%$ \\
Japón Nikkei & $9 \%$ & $4 \%$ & $14 \%$ & $2 \%$ \\
USA S\&P 500 & $27 \%$ & $10 \%$ & $33 \%$ & $2 \%$ \\
Argentina Merval & $3 \%$ & $0 \%$ & $16 \%$ & $1 \%$ \\
USA Dow Jones & $\mathbf{2 0} \%$ \\
Colombia IGBC & $\mathbf{1 2 0} \%$ & $\mathbf{1 2 4 \%}$ & $22 \%$ & $1 \%$
\end{tabular}

En el Cuadro $\mathrm{N}^{\circ}$ 3: Rendimientos Principales Índice Bursátil, se observa que la BVL fue la más rentable del mundo en el año 2006 en que el IGBVL alcanzó $187 \%$ en tanto el ISBVL Perú 15 fue $216 \%$ superior a la Bolsa de China (Shangai), que tuvo una rentabilidad de $138 \%$. Este desempeño superó largamente a la rentabilidad de la Bolsa de Colombia de 124\% que alcanzó en el año 2005.
Gráfico $\mathbf{N}^{\circ}$ 3. Evolución de la Balanza Comercial y proyecciones.

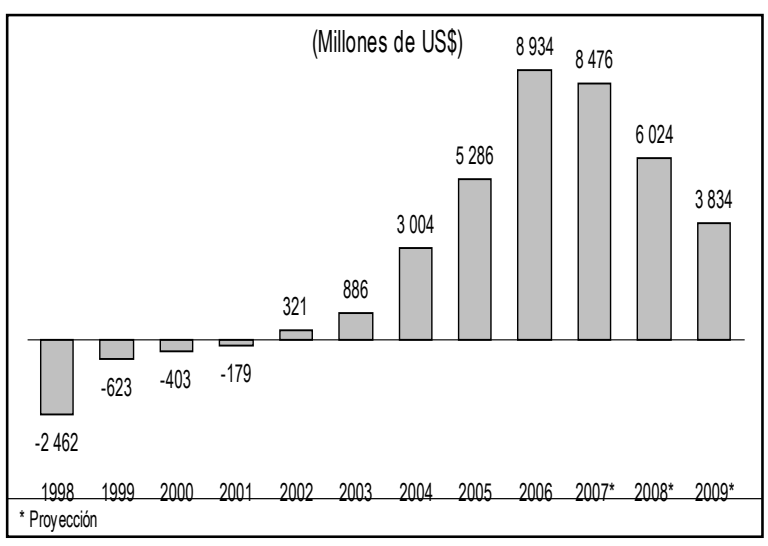

Al 13 de mayo del presente año, la BVL registró una rentabilidad de 48\% (IGBVL) descrita en el cuadro anterior; tendencia que varió por las volatilidades que la Bolsa ha venido experimentando, como lo ocurrido el 21 de noviembre cuando descendió a $31.30 \%$ (ver Cuadro $\mathrm{N}^{\circ}$ 4: Índices de Cotizaciones), no obstante haber alcanzado en el mes anterior niveles de $80 \%$. En tanto la Bolsa de China (Shangai) alcanzó la rentabilidad de $94.89 \%$ en el mismo período de tiempo durante el año.

Entre las acciones que han venido describiendo mayor rentabilidad en los años 2004-2005, se pueden destacar a las cinco primeras con mayores rendimientos que son el Banco Continental, 
Cuadro $\mathrm{N}^{\circ}$ 4. Índice de cotizaciones.

\begin{tabular}{llrrrrr}
\hline & Bolsa & \multicolumn{1}{c}{ Índice } & Var. día \% & Var. mes \% & Var. año \% & Var. \$ \% \\
\hline Lima & IGBVL & $\mathbf{1 6 , 9 5 5 . 4 8}$ & $-\mathbf{2 . 4 9}$ & $-\mathbf{2 1 . 8 5}$ & $\mathbf{3 1 . 6 0}$ & $\mathbf{4 0 . 9 4}$ \\
& ISBVL & $\mathbf{2 8 , 4 0 7 . 5 4}$ & -2.97 & -24.46 & $\mathbf{2 8 . 2 0}$ & $\mathbf{3 7 . 3 0}$ \\
& ISP-15 & $\mathbf{3 3 , 3 0 2 . 9 2}$ & $-\mathbf{2 . 9 7}$ & $-\mathbf{2 4 . 4 6}$ & $\mathbf{3 3 . 4 3}$ & $\mathbf{4 2 . 9 1}$ \\
& INCA & $\mathbf{8 8 . 1 9}$ & $-\mathbf{3 . 0 9}$ & $-\mathbf{2 0 . 3 8}$ & $\mathbf{n . d}$ & n.d \\
Sao Paulo & IBOVESPA & $60,581.54$ & -2.81 & -7.25 & 36.22 & 63.76 \\
México & IPC & $28,446.45$ & -2.08 & -9.58 & 7.55 & 5.81 \\
Buenos Aires & MERVAL & $2,216.15$ & -1.32 & -5.75 & 6.01 & 3.58 \\
Santiago & IGPA & $13,947.42$ & -1.37 & -9.11 & 12.72 & 16.92 \\
Colombia & IBVC & $11,157.23$ & -0.63 & 4.96 & -0.04 & 7.60 \\
Caracas & IBC & $37,821.96$ & -0.90 & -3.45 & -27.59 & -27.59 \\
Londres & FTSE 100 & $6,070.87$ & -2.50 & -9.68 & -2.41 & -7.38 \\
Frankfurt & Dax Index & $7,518.42$ & -1.47 & -6.24 & 13.97 & 28.19 \\
París & CAC 40 Index & $5,381.30$ & -2.28 & -7.98 & -2.90 & 9.23 \\
Madrid & IBEX 35 & $15,364.50$ & -.214 & -3.31 & 8.61 & 22.17 \\
Tokio & Nikkei 225 & $14,837.66$ & -2.46 & -11.35 & -13.86 & -5.57 \\
Hong Kong & Hang Seng & $26,618.19$ & -4.15 & -15.10 & 33.33 & 33.30 \\
Shanghai & Shanghai Composite & $5,214.23$ & -1.50 & -12.44 & 94.89 & 105.24 \\
New York & Dow Jones & $12,799.04$ & -1.62 & -8.12 & 2.70 & 2.70 \\
& NYSE Composite & $9,405.22$ & -1.78 & -8.79 & 2.91 & 2.91 \\
& NASDAQ Composite & $2,562.15$ & -1.33 & -10.39 & 6.08 & 6.08 \\
& & & & & & \\
\hline
\end{tabular}

Corporación Aceros Arequipa, Perubar Inv, Alicorp, Gloria Inv. I1, las mismas que han alcanzado una elevada rentabilidad como por ejemplo el Banco Continental que alcanzó $285 \%$ y Aceros Arequipa que llegó a $283 \%$ de rentabilidad acumulada del 2004 al 25 de octubre del 2005. La rentabilidad se puede apreciar en el Cuadro $\mathrm{N}^{\circ} 5$, en la columna de variación de lucratividad.

La posición anterior varía ante la importante presencia de las empresas mineras como consecuencia del alza de los precios de los minerales en los mercados internacionales, que conllevó a la obtención de elevada rentabilidad, siendo las de mayor preferencia por los inversores lo que impulsó el incremento de sus cotizaciones en Bolsa, produciendo mayor rentabilidad (variación de lucratividad), situación que se puede apreciar en los años 2005, 2006 y en lo que va del año 2007. Las empresas mineras más rentables se pueden apreciar en el Cuadro $\mathrm{N}^{\circ}$ 6: Acciones más rentables del IGBVL (2004-Mayo 2007). Tal es el caso de Volcán B que en el 2004 alcanzó $116 \%$ y en el 2006 se elevó a $763 \%$ de rentabilidad.

\section{ACERCA DE LA VOLATILIDAD}

Volatilidad se entiende como el cambio brusco en los precios de los distintos valores debido a las alteraciones de variables económicas, riesgo soberano, riesgo de tasa de interés, riesgo cambiario, riesgo político, etc. De acuerdo a estos parámetros,

Cuadro N. ${ }^{\circ}$ 5. Acciones más rentables dsel IGBVL (2004-octubre 2005).

\begin{tabular}{|l|c|c|c|c|c|}
\hline \multicolumn{1}{|c|}{ Acciones } & Precio de cierre & \multicolumn{4}{c|}{ Índice de lucratividad } \\
\hline \multicolumn{1}{|c|}{ IGBVL } & $21 / 10 / 2005$ & 2005 & $\%$ & 2004 & Rent. (var. de lucr.) \\
\hline Bco. Continental Corp & 5,50 & 212,40 & 112,40 & 181,20 & 284,47 \\
Aceros Arequipa & 1,58 & 108,60 & 8,60 & 353,00 & 283,36 \\
Perubar Inv. & 2,90 & 215,80 & 115,80 & 166,30 & 258,88 \\
Alicorp & 1,95 & 135,40 & 35,40 & 261,80 & 254,48 \\
Gloria Inv. I1 & $3,50-$ & 207,80 & 107,80 & 148,20 & 207,96 \\
\hline
\end{tabular}


Cuadro N $^{\circ}$. Acciones más rentables del IGBVL (2004-mayo 2007)

\begin{tabular}{|c|c|c|c|c|c|c|c|c|c|}
\hline \multirow{2}{*}{ Acciones IGBVL } & \multirow{2}{*}{$\begin{array}{c}\text { Precio } \\
\text { de cierre } \\
30 / 05 / 2007\end{array}$} & \multicolumn{8}{|c|}{ Índice de lucratividad } \\
\hline & & $\begin{array}{c}30 / 05 / 20707- \\
29 / 12 / 2006 \\
\end{array}$ & $\%$ & 2006 & $\%$ & 2005 & $\%$ & 2004 & $\%$ \\
\hline Volcán "B" & $\begin{array}{c}12,95 \\
474\end{array}$ & $\begin{array}{l}177,2 \\
1374\end{array}$ & $\begin{array}{l}77,2 \\
374\end{array}$ & $\begin{array}{l}862,7 \\
1317\end{array}$ & $\begin{array}{r}763 \\
317\end{array}$ & 91,9 & $-8,1$ & 215,8 & 116 \\
\hline Peru Cooper & 4,74 & 137,4 & 37,4 & 131,7 & 31,7 & & & & \\
\hline Cerro Verde & 19,79 & 137,9 & 37,9 & 337,7 & 238 & 124,3 & 24,3 & 136,9 & 36,9 \\
\hline Atacocha Inv. & 9,5 & 162,2 & 62,2 & 464,4 & 364 & 145,6 & 45,6 & 203,9 & 104 \\
\hline Morococha Inv. & 5,45 & 82,3 & $-17,7$ & 1103,3 & 1003 & 116,6 & 16,6 & 233,4 & 133 \\
\hline
\end{tabular}

Gráfico $\mathrm{N}^{\circ}$ 4. Acción muy volátil.

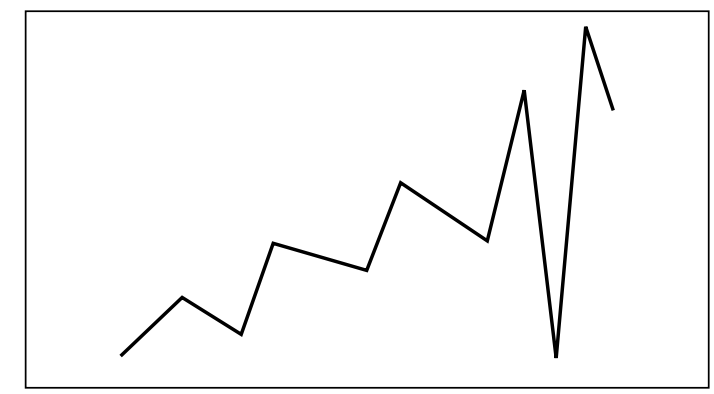

Gráfico $\mathbf{N}^{\circ} 5$. Acción poco volátil.

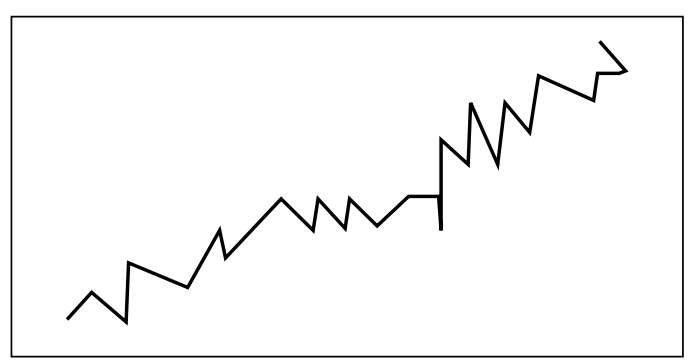

se habla de un valor de "alta volatilidad" cuando éste puede mostrar variaciones importantes (a la alza o a la baja), dependiendo de las condiciones imperantes de los mercados (ver Gráficos N. ${ }^{\circ} 4$ y 5). La volatilidad de una acción hace referencia a las oscilaciones que presenta su cotización.

El grado de oscilación que presenta una acción, se mide con el indicador denominado Beta, que compara la volatilidad de la acción que se presenta en la Bolsa en su conjunto. Comentaremos diferentes comportamientos de acciones a través del Beta:

- BETA > 1. Acción de elevada volatilidad, varía más que el mercado. Ejemplo: una acción con una Beta del 1.5 significa que históricamente ha oscilado un $50 \%$ más que el mercado, tanto en subida como en bajada. Si el mercado ha subido un $10 \%$, esta acción ha subido un $15 \%$, y si el mercado ha bajado un $10 \%$, esta acción lo ha hecho en un $15 \%$.
- $\quad B E T A=1$. Acción con la misma volatilidad que el mercado. Ejemplo: si el mercado ha subido un $10 \%$, esta acción ha subido otro $10 \%$, y si el mercado ha bajado $105 \%$, esta acción ha bajado lo mismo.

- BETA<1. Acción de poca volatilidad, varía menos que el mercado. Ejemplo: una acción con una Beta de 0.3 significa que dicha acción ha oscilado históricamente un $30 \%$ de lo que lo ha hecho el mercado: si el mercado ha subido un $10 \%$, esta acción ha subido un $3 \%$; si el mercado ha bajado un 10\%, esta acción ha bajado un $3 \%$.

- BETA<0. Es una situación poco habitual pero que se puede presentar. Significa que la acción varía en sentido contrario a lo que hace el mercado: si el mercado sube la acción baja, y viceversa.

Se podrían distinguir otros casos:

- Intervalo - $1<$ BETA < 0. La acción varía en sentido contrario al del mercado pero con menor intensidad. Ejemplo: una acción con una Beta de - 0.4 significa que si el mercado ha subido un $10 \%$ esta acción ha bajado un $4 \%$, y si el mercado ha bajado un $10 \%$, esta acción ha subido un $4 \%$.

- $\mathrm{BETA}=-1$. La acción oscila igual que el mercado pero en sentido contrario.

- BETA<-1. La acción oscila más que el mercado y en sentido opuesto.

\section{Volatilidad de la Bolsa de Valores de Lima}

Como expusimos, la Bolsa de Valores es un mercado expuesto al riesgo; por tanto se ve afectado por los efectos externos y crisis de los mercados. Su comportamiento lo podemos apreciar en las variaciones del IGBVL, nivel que está fuertemente ligado a la evolución de los principa- 
les mercados internacionales, especialmente a la Bolsa de New York, cuyo principal índice es el Dow Jones. Dado que ese país está sufriendo una crisis en el mercado de hipotecas de alto riesgo se ha generado un gran temor entre los inversionistas por una posible desaceleración de la economía, lo que explica la volatilidad del mercado en los últimos meses, como apreciamos en el Gráfico $\mathrm{N}^{\circ}$ 6 donde vemos la comparación de los índices de la Bolsa de Lima con la Bolsa de Nueva York entre junio 2007 y octubre 2007, observándose que el Índice de Dow Jones es poco volátil mientras que IGBVL señala que tiene una alta volatilidad.

Durante el presente año han ocurrido varios sucesos importantes que afectaron a la Bolsa de Lima (Ver Gráfico $\mathrm{N}^{\circ} 7$ ). Así en enero, el grupo Interbank realizó la transferencia del íntegro de sus acciones de Interbank e Interseguro a favor de su subsidiaria Intergroup Financial Services Corp. Como se dio entre personas jurídicas del mismo grupo no fue necesario realizar una oferta pública de adquisición.
El 30 de mayo el Índice General cayó cerca del 8\%, lo que representó la mayor caída en los últimos doce años debido a la caída de la Bolsa de China, como consecuencia de la decisión de las autoridades chinas de aumentar el Impuesto a las Transacciones Accionarias del $0.1 \%$ al $0.3 \%$. En setiembre la FED bajó la tasa de interés de 5.25 a $4.75 \%$ para aliviar la crisis financiera y económica de Estados Unidos y el gran aumento del flujo de capitales hacia el Perú. Además el 31 de octubre la FED redujo nuevamente la tasa de interés referencial a $4.5 \%$ pero no se dio un efecto positivo por dos razones: los inversionistas ya habían internalizado la decisión de la FED, por lo que al recibir el anuncio realizaron una toma de ganancias generalizada, y segundo, porque los inversionistas no consideran que el recorte de la tasa de interés sea suficiente para solucionar la crisis crediticia en Estados Unidos. Las expectativas estarían condicionadas al comportamiento de los metales y a los indicadores que se publiquen sobre la economía de Estados Unidos.

Gráfico Nº6. Comparación de los índices de la Bolsa de Lima y la Bolsa de Nueva York.

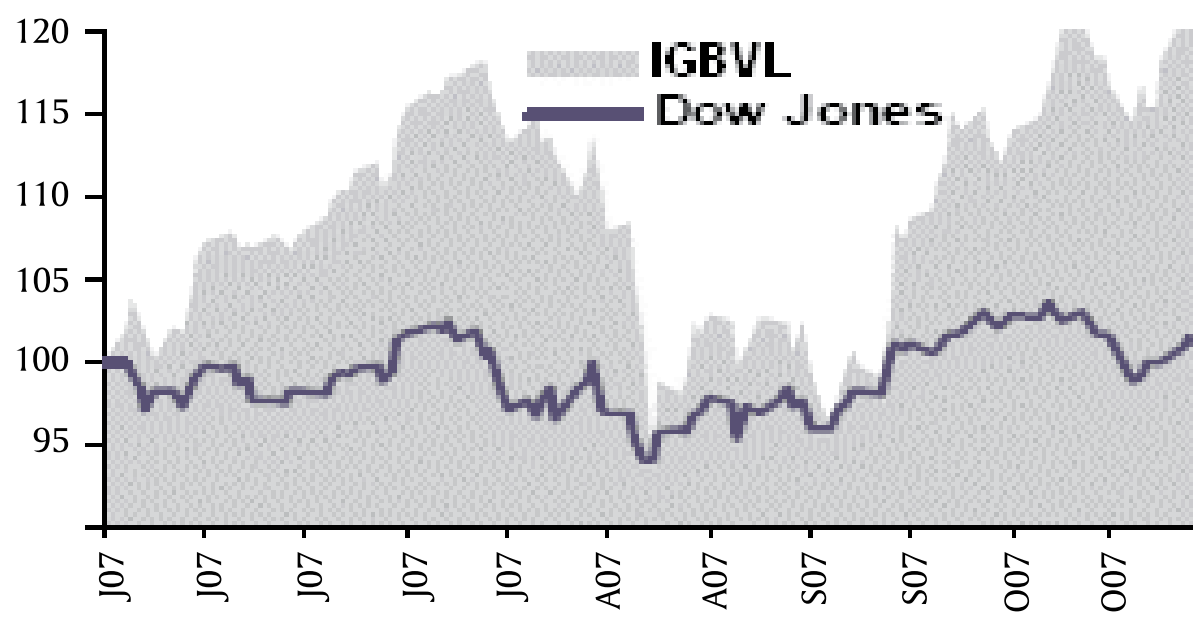

Fuente: Bloomberg / Elaboración: Análisis - BCP 
Gráfico $\mathbf{N}^{\circ}$ 7. Sucesos importantes que afectaron a la Bolsa de Lima.

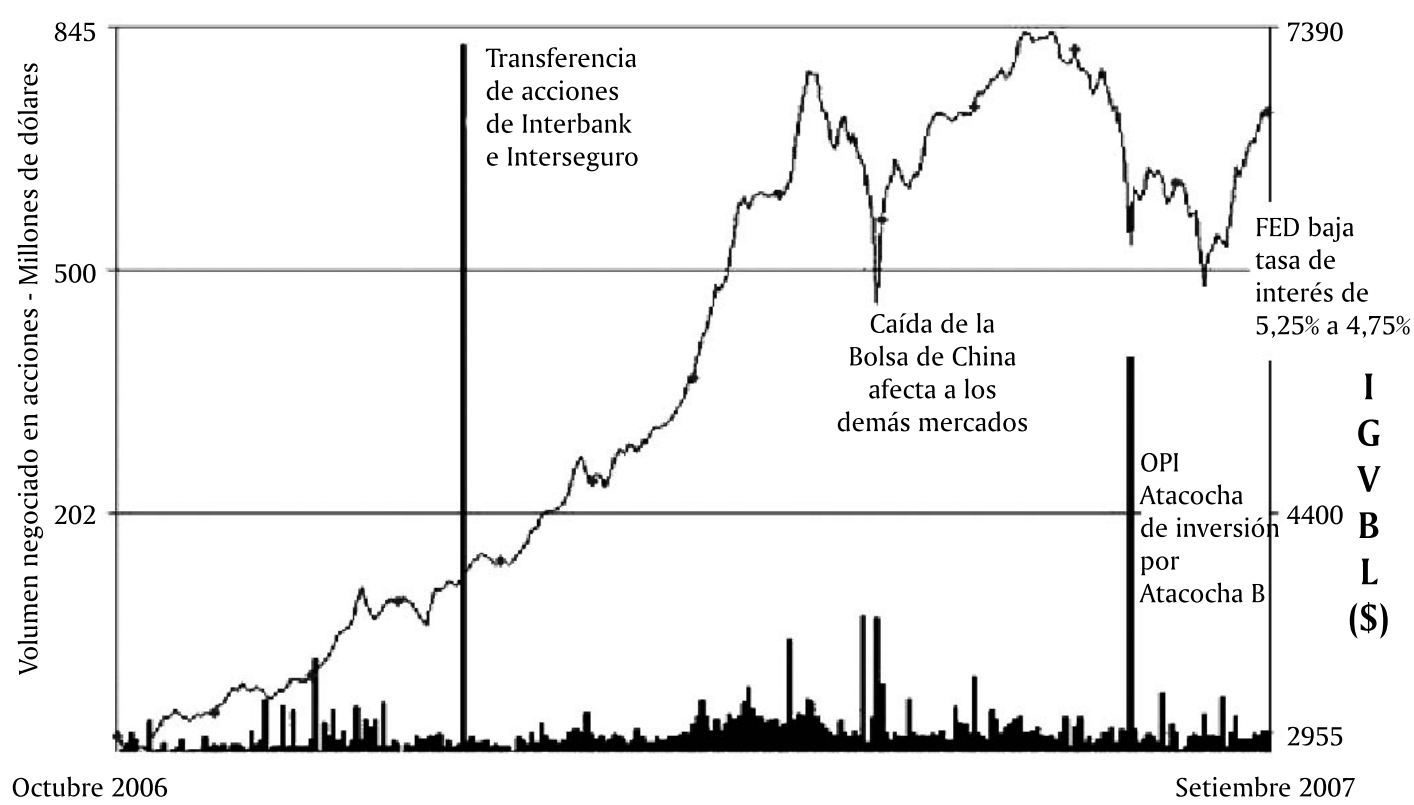

Si tomamos en cuenta las variables y conceptos antes mencionados podemos entender el qué, el cómo y el por qué de invertir en bolsa, información esencial en las decisiones de inversión; es decir, la decisión de invertir en Bolsa debe estar enmarcada en un horizonte de largo plazo, entendiéndose que los recursos asignados a este mercado no deben ser comprendidos en las necesidades prioritarias del inversor.

\section{BIBLIOGRAFÍA}

WESTON Y COPELAND. (1999). Finanzas en administración. México, McGraw Hill.

BREALEY Y MYERS. (1998). Principios de finanzas corporativas. México, Prentice \& Hall.

MARTÍNEZ ABASCAL, E. (1998). Invertir en Bolsa. España. Serie McGraw Hill-IESE.

VAN HORNE. (1999). Fundamentos de administración financiera. México, McGraw Hill.

WESTON Y BRIGHAM. (1996). Fundamentos de administración financiera. México, McGraw Hill.

EMERY Y FINNERTY (2002). Administración financiera corporativa. México, Prentice \& Hall.

BOLSA DE VALORES DE LIMA. (2004). Boletín Diario. Lima, BVL.

BOLSA DE VALORES DE LIMA. (2005). Vademécum Bursátil. Lima, BVL.

BOLSA DE VALORES DE LIMA. (2006). Informes Bursátiles. Lima, BVL.

MARCASE, Robert. (2004). Operaciones Bancarias en las Américas. ASBANC.

Informes y estadísticas del BCRP, CONASEV, BVL, SBS, MEF, INEI.

\section{PÁGINAS WEB}

www.bvl.com.pe

www.nyse.com

www.patagon.com

$w w w . v i a i n v e r s i o n e s . c o m$

www.conasev.gob.pe

www.sbs.gob.pe

www.bcrp.gob.pe 